\title{
Introduction
}

\section{Evolving trends in movement disorder surgery}

\author{
Sameer A. Sheth, M.D., Ph.D., ${ }^{1}$ Emad N. Eskandar, M.D. ${ }^{2}$ \\ AND MARK A. LiKer, M.D.
}

\footnotetext{
${ }^{1}$ Department of Neurosurgery, NewYork-Presbyterian Hospital, Columbia University, New York, New York; ${ }^{2}$ Department of Neurosurgery, Massachusetts General Hospital, Harvard Medical School, Boston, Massachusetts; and ${ }^{3}$ Department of Neurosurgery, Keck Hospital, University of Southern California, Los Angeles, California

Movement disorder surgery has evolved significantly in the past few decades. Stereotactic brain lesion procedures were the mainstay of surgical treatments for movement disorders for much of the latter part of the previous century. Deep brain stimulation (DBS) began increasing in popularity in the last decade of the 20th century and has had tremendous success in treating these disorders, with more than 100,000 patients having undergone implantation.
}

In this issue of Neurosurgical Focus, we concentrate on emerging trends in movement disorder surgery. The issue begins with an update of the current understanding of the mechanism of DBS and a review of current clinical indications for neurological and psychiatric disorders as well as emerging indications (Karas et al.). Following this review are articles considering specific aspects of DBS surgery, including a Nationwide Inpatient Sample database study of DBS outcomes and costs (Sharma et al.), an article on novel electrode recording techniques (Jonker et al.), and articles on DBS for Tourette's syndrome (Rotsides et al.) and pediatric dystonia (Olaya et al.). Also in this issue are an article describing the persistent utility of lesion procedures in the DBS era (Bulluss et al.) and an article on selective dorsal rhizotomy (Gump et al.), an effective peripheral nervous system treatment for certain spasticity disorders.

(http://thejns.org/doi/abs/10.3171/2013.9.FOCUS13392)

\section{Disclosure}

The authors report no conflict of interest.

Please include this information when citing this paper: DOI: 10.3171/2013.9.FOCUS13392. 